Journal Club

Editor's Note: These short, critical reviews of recent papers in the Journal, written exclusively by graduate students or postdoctoral fellows, are intended to summarize the important findings of the paper and provide additional insight and commentary. For more information on the format and purpose of the Journal Club, please see http://www.jneurosci.org/misc/ifa_features.shtml.

\title{
What Is Driving Inhibition-Related Activity in the Frontal Lobe?
}

\author{
Franz-Xaver Neubert ${ }^{1}$ and Miriam C. Klein ${ }^{2}$ \\ ${ }^{1}$ Department of Experimental Psychology, University of Oxford, Oxford OX1 3UD, United Kingdom, and 25obell Department of Motor Neuroscience and \\ Movement Disorders, Institute of Neurology, University College London, London WC1N 3BG, United Kingdom \\ Review of Chikazoe et al.
}

One of the main challenges of cognitive neuroscience is to refine existing concepts and models of human behavior so that they are grounded in and in agreement with brain processes observed during a particular behavior. Inhibition has been of particular interest. In a physiological context, inhibition refers to the causal influence exerted by region $\mathrm{A}$ on region $\mathrm{B}$, whereby region A decreases excitability and output firing of region B. In contrast, in cognitive models of behavior, inhibition is the suppression of previously activated cognitive contents or processes. Although it has been suggested that brain areas implement cognitive control by exerting inhibitory physiological influence over other brain areas (Aron et al., 2003), it is still unclear to what extent inhibition on a behavioral level can be related to physiological mechanisms of inhibition. Here we focus on the frontal lobe mechanisms of behavioral inhibition. This type of inhibition is a vital part of human behavior because it allows flexible adaptation to changing environments, such as the clearing of irrelevant action plans or attention. It is often studied in the motor

Received Feb. 9, 2010; revised March 9, 2010; accepted March 10, 2010.

This work was funded by the Wellcome Trust (M.C.K.) and the German National Academic Foundation (F.X.N.).

Correspondence should be addressed to Miriam C. Klein, Sobell Department of Motor Neuroscience and Movement Disorders, UCL Institute of Neurology, Box 146, 33 Queen Square, London WC1N 3BG, United Kingdom. E-mail:m.klein@ucl.ac.uk.

DOI:10.1523/JNEUROSCI.0745-10.2010

Copyright $\odot 2010$ the authors $\quad 0270-6474 / 10 / 304830-03 \$ 15.00 / 0$ system, because sudden cancellations of movement plans occur frequently in everyday life and they can be easily controlled in an experimental setup.

The excitability and output firing of the primary motor cortex (M1), the area that finally enables movements, depends on a balance of M1 internal inhibitory and excitatory mechanisms, and these in turn depend on the behavioral context. For example, M1 excitability decreases in situations with higher uncertainty about which of multiple potential actions is required (Bestmann et al., 2008). To understand the mechanisms of inhibitory control, we therefore need to understand how other areas feeding into $\mathrm{M} 1$, such as premotor and prefrontal regions, influence M1 excitability and output when a movement needs to be inhibited.

Thus far, inhibition from a cognitive perspective has mainly been studied using stop-signal tasks. Such tasks have two types of trials. In go trials, participants are asked to make a speeded response to a visual cue. In no-go trials, however, the go signal is, after a short delay, followed by a stop signal (e.g., a tone or visual cue) instructing the participant to withhold their response.

Studies using stop-signal paradigms have highlighted the importance of several frontal brain regions in the control of response inhibition, in particular the right inferior frontal gyrus (rIFG) and the presupplementary motor area (pre-SMA). Lesions to rIFG and pre-SMA interfere with the ability to stop a planned movement, and the degree of damage to the rIFG is negatively correlated with performance in a stop-signal task (Aron et al., 2003; Nachev et al., 2007). Imaging studies reported activity in pre-SMA and IFG associated with stopping processes (Aron and Poldrack, 2006; Li et al., 2006). Those participants who were better at inhibiting responses showed more activity in rIFG and pre-SMA and higher white matter density in tracts connecting rIFG with M1 (Li et al., 2006; Forstmann et al., 2008). Finally, repetitive transcranial magnetic stimulation to both rIFG and pre-SMA transiently impaired performance on go/ no-go tasks (Chambers et al., 2006). These and other findings suggest that both rIFG and pre-SMA play a role in response inhibition. Their distinct contributions in inhibitory control have only recently started to become clear. It was suggested that rIFG exerts inhibitory control over motor outputs (i.e., decreases physiologically excitability of M1), while pre-SMA might facilitate changing planned movements and resolving conflict between competing actions (Aron and Poldrack, 2006; Mars et al., 2009).

What remains unknown is how other contextual variables influencing M1 excitability, such as the uncertainty with which a movement is prepared and the likelihood that the movement will need to be inhibited, interact with inhibitory control. In studies using standard stop-signal tasks, participants have a certain expecta- 
tion of a stop signal on each trial. Therefore, go responses are always associated with some level of uncertainty, and processes related to inhibitory control and the resolution of conflict between different potential actions (moving versus stopping) cannot be fully separated. A recent study by Chikazoe et al. (2009) published in The Journal of Neuroscience therefore used a modified stop-signal task that allowed some control over the uncertainty involved in making a go response. The study thereby offers the opportunity to bridge findings from studies on inhibitory control with concepts such as uncertainty that are increasingly the focus of research into decision making (Rushworth et al., 2009).

Chikazoe et al. (2009) used a standard stop-signal task with one novel feature: apart from the common "uncertain" go signals, which were as usual followed by a stop signal in a small percentage of trials $(20 \%)$, there was also a new trial type in which a differently colored go cue signaled a "go" with $100 \%$ certainty ("certain go"), i.e., it was never followed by a stop signal. This enabled the authors to investigate the effect of the certainty with which the movement was prepared, and the possible "precautionary" preparation of the potential need to inhibit a response.

The difference between certain and uncertain go trials was examined (1) behaviorally by comparing reaction times of both trial types and (2) by using functional magnetic resonance imaging ( $\mathrm{fMRI}$ ) to compare the blood oxygenation level-dependent (BOLD) signal in the two conditions. Brain regions more active for uncertain compared to certain go trials were identified (referred to by the authors as "preparation" contrast) and compared to those identified in the comparison between stop and uncertain go trials ("inhibition" contrast). Comparisons in the form of conjunction and disjunction analyses between the activation patterns established by these two initial contrasts were also performed. Finally, the correlation between behavioral reaction times and BOLD signals was determined.

It was found that in situations when a go signal might be followed by a stop signal ("uncertain go"), participants responded on average 105.5 ms slower than on certain go trials. The areas more active for uncertain compared to certain go cues ("preparation" contrast) were the pre-SMA, inferior frontal junction (IFJ), and insula. Compared to uncertain go trials, a successful inhibition was associated with more activity in the pre-SMA, insula, and, among other areas, the posterior IFG ("inhibition" contrast). The conjunction analysis identified which areas were active in both contrasts: pre-SMA, IFG, and insula. Interestingly, only the right posterior part of IFG was more active during stop versus uncertain go but not uncertain versus certain go (disjunction analysis) [Chikazoe et al. (2009), their Fig. 3].

Chikazoe et al. (2009) concluded that higher reaction times in uncertain compared to certain go trials were explained by additional preparation for a potentially necessary response inhibition in uncertain go trials. Similarly, imaging findings were interpreted as showing brain activity reflecting "preparation to inhibit." It was argued that this preparation to stop could facilitate the neuronal processes needed for subsequently implementing the inhibition itself by recruiting the same areas. This interpretation was supported by the finding that subjects who showed greater reaction time increases on uncertain go trials were the same subjects who had less inhibition-related activity [Chikazoe et al. (2009), their Fig. 4B]. The authors concluded that pre-SMA and IFJ might play a role in preparing inhibition in uncertain go trials to then facilitate fast and more efficient stopping in the event that a stop cue is presented.

Chikazoe et al. (2009) introduced the novel concept of "prepared inhibition" in the context of stop-signal tasks, according to which, in "uncertain go" trials, inhibition is prepared in anticipation of an upcoming stop cue. Thus far in the literature, however, inhibition has mostly been thought of as a process that helps one rapidly adjust behavior in situations when predictions are violated. But then to prepare inhibition would effectively mean being able to predict these prediction violations. We would like to discuss some alternative interpretations and possible implications of this framework.

First, instead of interpreting activity in areas reported to be more active in uncertain compared to certain go trials (such as IFJ and pre-SMA) as preparation-related activity, it could be explained in terms of task switching: switching from "certain go" to "uncertain go" would activate the respective task set representations. IFJ and pre-SMA have previously been associated with task set representation and implementation. Hence the idea that preparation to inhibit expedites inhibition could be more intuitively explained as effects of task switching.

Second, pre-SMA has previously been associated with conflict resolution and adjustment of cognitive control (Mars et al.,
2009). The "preparation" contrast analyzed by Chikazoe et al. (2009) does not rule out such an interpretation. Areas active in uncertain compared to certain go trials could also be responding to the higher degree of conflict and uncertainty present in these trials. Similarly, they could respond to higher levels of attention or the adjustment of a speedaccuracy trade-off. All these interpretations would also explain why subjects responded more slowly in uncertain compared to certain go trials.

Third, it cannot be ruled out that some truly inhibitory processes might also be present in uncertain go trials, possibly to prevent early and incautious responses.

Finally, the results of Chikazoe et al. (2009) could be placed within a more general framework of a "proactive" brain, which has been shown to explain important aspects of decision making and action selection (Rushworth et al., 2009). It suggests that the brain constantly makes predictions about future events, appropriate behavior, rewards and outcomes, and risks, and that brain activity is largely driven by the uncertainty related to these predictions and by prediction violations. According to this view, predictions generated at higher levels on the basis of past experiences and explicit knowledge (such as rules) could influence lower-level processing of sensory information and motor output. In this context, the "uncertain go" trials of Chikazoe et al. (2009) might recruit the network of frontal brain areas to subsequently adjust processing in lowerlevel sensory-motor circuits and to inhibit excitability of the motor system in a situation with higher uncertainty. Hence the areas recruited by "uncertain go" trials (pre-SMA, IFJ, etc.) might actually be involved in the rapid updating of predictions about future inputs and appropriate responses (i.e., the currently active task schema).

To further explore inhibition within such a conceptual framework, it would be helpful to extend the authors' novel task design in a future study by using "uncertain go" trials with varying degrees of uncertainty (parametric modulation). Sometimes the probability of a stop signal occurring might be $10 \%$, at other times $20 \%, 30 \%$, etc. One would expect preparation for action to decrease with increasing uncertainty, while preparation to inhibit should (maybe) increase. The level of uncertainty could be controlled for independent of the level of action/inhibition preparation by reversing the proportion of go/no-go trials (i.e., $80 \% / 20 \%$ vs $20 \%$ / 
$80 \%)$. Preparation and uncertainty could then be modeled separately in the fMRI data, and their trial-by-trial fluctuations could be related to brain activity. This would allow testing of further predictions about the interactions of such contextual variables with the processes involved in response inhibition.

In summary, the study by Chikazoe et al. (2009) provides a platform that will motivate further studies toward a better understanding of the links between action inhibition and theories of cognitive control, decision making, and motor planning.

\section{References}

Aron AR, Poldrack RA (2006) Cortical and subcortical contributions to Stop signal response inhibition: role of the subthalamic nucleus. J Neurosci 26:2424-2433.

Aron AR, Fletcher PC, Bullmore ET, Sahakian BJ, Robbins TW (2003) Stop-signal inhibition disrupted by damage to right inferior frontal gyrus in humans. Nat Neurosci 6:115-116.

Bestmann S, Harrison LM, Blankenburg F, Mars RB, Haggard P, Friston KJ, Rothwell JC (2008) Influence of uncertainty and surprise on human corticospinal excitability during preparation for action. Curr Biol 18:775-780.

Chambers CD, Bellgrove MA, Stokes MG, Henderson TR, Garavan H, Robertson IH, Morris AP, Mattingley JB (2006) Executive "brake failure" following deactivation of human frontal lobe. J Cogn Neurosci 18:444-455.

Chikazoe J, Jimura K, Hirose S, Yamashita K, Miyashita Y, Konishi S (2009) Preparation to inhibit a response complements response inhibition during performance of a stopsignal task. J Neurosci 29:15870-15877.

Forstmann BU, Jahfari S, Scholte HS, Wolfensteller U, van den Wildenberg WP, Ridderinkhof KR
(2008) Function and structure of the right inferior frontal cortex predict individual differences in response inhibition: a model-based approach. J Neurosci 28:9790-9796.

Li CS, Huang C, Constable RT, Sinha R (2006) Imaging response inhibition in a stop-signal task: neural correlates independent of signal monitoring and post-response processing. J Neurosci 26:186-192.

Mars RB, Klein MC, Neubert FX, Olivier E, Buch ER, Boorman ED, Rushworth MF (2009) Short-latency influence of medial frontal cortex on primary motor cortex during action selection under conflict. J Neurosci 29:69266931.

Nachev P, Wydell H, O’Neill K, Husain M, Kennard C (2007) The role of the pre-supplementary motor area in the control of action. Neuroimage 36 [Suppl 2]:T155-T163.

Rushworth MF, Mars RB, Summerfield C (2009) General mechanisms for making decisions? Curr Opin Neurobiol 19:75-83. 